\title{
Professional standards in failure analysis and expert reports
}

\author{
D.F. Williams \\ Department of Clinical Engineering, University of Liverpool, P.O. Box 147, Liverpool L69 3BX, UK
}

\section{Synopsis}

During the last decade, the regulatory environment associated with the applications of medical devices has changed radically. In addition, the whole area has become embroiled in a mass of litigation concerning the responsibilities for any failure of a device to perform to required or anticipated standards. Although these two aspects are quite different in the context of the administration of law, they do have many similarities. Both are concerned with the delivery of optimal device performance to the patient, in the former case through laws which regulate their manufacture and sale, in the latter case through considerations of tort with respect to any deficiencies in performance.

In practice, however, there is one very important underlying principle that is common to both these manifestations of the medical device legal framework and that is the lack of objectivity in most aspects of the measurement of device performance and the need to rely upon subjective opinion. This presentation is concerned with the professional standards of those who provide these opinions. The need for such comment arises from the considerable pressures placed upon experts whose views are respected by regulatory agencies and the judiciary but where the financial consequences of their decisions can be so great.

Dealing first with the question of litigation, it is widely known that 'failures' of implantable medical devices have resulted in large numbers of individual claims for compensation and several multi-billion dollar class actions. If patients stand to receive hundreds of thousands to millions of dollars if they can convince a judge or jury that they have been injured by a device, clearly they and their attorneys need to present their case in the most effective way. How much better it is to have an expert who can testify that, in his or her opinion, the failure to perform as anticipated was a consequence of negligence by the manufacturer. It is so easy under such circumstances to lose some objectivity and present cases stronger than they are or to ignore the benefits and/or difficulties associated with procedures.

In the view of this author, it is perfectly correct and indeed necessary for all those involved in the delivery of health care, including the provision of any commercial products, to be held accountable for their actions and for patients who are clearly and significantly disadvantaged as a result of any treatment to be compensated in some way. In extreme cases of negligence or malicious actions, the questions of punitive damages are rightly raised. However, the use of implantable devices has to take into account the factors of risk assessment and the full benefits of procedures that perform satisfactorily 
in the vast majority of cases have to be weighed against the lack of benefit if that satisfaction is not obtained.

This calls into question the whole concept of failure. What is meant by the failure of an implanted device? If the femoral component of a total hip replacement fractures two years after implantation, the device and therefore the procedure have clearly failed in the sense that the patient no longer has the function that he or she had a right to expect. Nobody would accept a hip replacement if they were told that it would only last two years at which time it would fracture. It hardly needs an expert to diagnose a fractured prosthesis, but it may need an expert to explain the failure and to offer an opinion as to whether it was a manufacturing defect, a surgical error or even patient abuse. Or perhaps it was none of these but rather a fact of life that cannot easily be explained. The expert's examination of all of the factors associated with this clear failure is clearly of great significance, and whilst it will encompass some objective assessment of the technological aspects, it will almost certainly also include some subjective opinions.

If another patient received a similar prosthesis and this lasts twenty years before it fractures, do we consider that as a failure? It could be argued that materials science has advanced sufficiently well for alloys of perfectly adequate fatigue life to be chosen for the stems of these prostheses such that they should never fracture. Alternatively, it could be argued that a device that rescued the patient from extreme pain and a disabling lack of mobility and gave that patient twenty years of high quality life which he would otherwise not have had, could not possibly be described as a failure, even if it did fracture at twenty years. After all, the patients own joints, developed after millions of years of evolution, failed to perform to specification; how can we, after only a few decades of development, provide guaranteed life-time successful performance in such situations? A very considerable responsibility rests with the experts who conduct the failure analysis to include all such factors in their assessment of responsibility.

These responsibilities become even more onerous and problematic when the views of an expert are translated into the legal framework of court testimony and deposition. Scientists of high quality frequently become trapped by the experienced attorney and say things they never meant to say and even say outrageous things in the heat of the courtroom. It has to be said that a number of scientists of lower quality find that they are able to handle this situation better than others and it is their style and their presentation that are more effective than their views. The professional litigation expert witness is here and many judgements are based on their courtroom skills rather than on the scientific and technical skills.

As far as the regulatory aspects are concerned, there is nothing new in the use of expert reports in submissions of files and dossiers to support applications for product registration. As the whole area of regulatory approval for devices in Europe is changing, the role of expert reports and technical assessments has yet to evolve. However, the scenario of submissions being considered by Notified Bodies, whose expertise lies solely within quality assurance procedures and not in the technical areas of medical devices, suggests that the use of expert assessors will be crucial. Again, bearing in mind the very considerable financial consequences of the approval process and also the fact that those involved in the regulatory control are themselves profit making organisations, this would point to the need for considerable professionalism and objectivity in this context.

The present conference is concerned with post-market surveillance with relevance to implant and patient tracking and device retrieval. All of the above comments from both regulatory and legal ends apply here, since surveillance of the performance of devices is an obvious common denominator. 


\section{D.F. Williams}

Professor D.F. Williams is Head of the Department of Clinical Engineering at the University of Liverpool, UK. He was initially trained as a metallurgist and has worked for the last 28 years in the area of biomaterials and implantable medical devices. He has authored or edited over 30 books and published over 220 scientific papers. His own research work has concentrated on the biocompatibility of biomaterials. He has widespread experience in advising the medical device industry on matters of biological testing and the development of biomaterials and advises many governmental bodies on policies and priorities for the funding of medical research. 